\title{
Malaria in pregnancy
}

\author{
P Soma-Pillay FCOG (SA) Cert (Maternal and Fetal Medicine) SA and A P Macdonald FCOG (SA) FRCOG
}

Steve Biko Academic Hospital - Obstetrics \& Gynaecology, Private Bag X169, Pretoria, Gauteng 0001, South Africa

\begin{abstract}
Summary: Malaria is a complex parasitic disease affecting about 32 million pregnancies each year in sub-Saharan Africa. Pregnant women are especially susceptible to malarial infection and have the risk of developing severe disease and birth complications. The target of Millennium Development Goal 6 is to end malaria deaths by 2015. Maternal and perinatal morbidity and mortality due to malaria may be reduced by implementing preventive measures, early diagnosis of suspected cases, effective antimalarial therapy and treatment of complications.
\end{abstract}

Keyword: infectious diseases

\section{INTRODUCTION}

Malaria infection in pregnancy complicates about 32 million pregnancies each year in endemic areas in sub-Saharan Africa and can be life-threatening for both the mother and child. ${ }^{1}$ The severe burden of disease has led to a global fight to control malaria. The target of the Millennium Development Goal 6 is to end malaria deaths by 2015 and international funding for malaria control has risen sharply in the last decade. The World Malaria Report states that by the end of 2010, insecticide-treated mosquito nets would have been delivered to sub-Saharan Africa to protect about 580 million people - this would protect $76 \%$ of the population at risk. ${ }^{2}$ More than 75 million people have received protection from indoor residual spray. Mosquito sprays containing permethrin or pyrethroids kill resting and flying mosquitoes.

Despite these interventions, there needs to be a greater focus on intervention for prevention of malaria in pregnancy. Van Eijk et al. $^{3}$ have found that only $17 \%$ of pregnant women in sub-Saharan Africa are protected with insecticide-treated bed nets. Disparities also exist in levels of care. A woman in Zambia is 25 times more likely to receive interventions to prevent malaria than a woman in Angola. ${ }^{4}$ Logistical challenges of reaching remote regions, lack of infrastructure and poverty contribute to complicated health service delivery in developing countries.

\section{PATHOPHYSIOLOGY}

The female Anopheles mosquito is responsible for transmission of malaria in humans. Asexual replication and maturation of Plasmodium occur in the host's liver and later within the red blood cells. Further division of the trophozoite causes rupture of the red blood cells, releasing merozoites, haemoglobin, malaria pigments and toxic cellular debris. This causes the characteristic fever patterns of the different malaria parasites.

Correspondence to: Priya Soma-Pillay

Email: priya.somapillay@up.ac.za
The typical cyclic fever occurs every 48 hours for Plasmodium vivax, Plasmodium ovale and Plasmodium falciparum and every 72 hours for Plasmodium malariae. Organ damage is believed to be caused by clogging of the microcirculation and the release of various cytokines.

Complications of malaria in pregnancy include maternal anaemia, low birth weight, prematurity and increased perinatal mortality. ${ }^{5}$ Malarial infection during pregnancy is characterized by the marked accumulation of parasites in the intervillous spaces of the placenta, where infected red blood cells adhere to the endothelial lining. This adhesion or sequestration is mediated through a specific parasite-encoded variant surface antigen (VSA) that is found on the membrane of the infected red blood cell. ${ }^{6}$ The VSA binds to chondroitin sulphate A on the syncytiotrophoblast lining the intervillous space. The sequestration causes the release of inflammatory leukocytes, which causes necrosis of the placental tissue.

Multigravid women in endemic areas are somewhat protected from placental malaria and this may be the result of maternal antibodies preventing cytoadhesion of the parasite to the placenta. ${ }^{7,8}$ This protection is lost when women move away from endemic regions and they are therefore at risk if they move back again after some time away.

\section{PREVENTION OF MALARIA IN PREGNANCY}

\section{Short-term travellers}

Pregnant women should be advised against travelling to malarial areas during pregnancy. They should be informed of the serious complications of malaria in pregnancy and the effects of antimalarial drugs. If a patient develops malarial symptoms, she should seek urgent medical care. If travel is unavoidable, prophylactic antimalarial medication should be taken. Chloroquine, proguanil and mefloquine are recommended in pregnancy. The choice of medication depends on the area that the woman is visiting. It is also important 
that the medication is prescribed appropriately for the travel date and duration.

These chemoprophylactic agents should be combined with other measures to prevent mosquito bites:

- Remaining indoors between dusk and dawn;

- Wearing long trousers and long sleeves;

- Applying insect repellent to exposed skin;

- Sleeping in a well-screened or air-conditioned room or under an insecticide treated bed-net.

\section{Prevention in endemic areas}

The World Health Organization (WHO) recommends three interventions for prevention and control of malaria in pregnancy in endemic areas: ${ }^{9}$

- Intermittent preventive treatment;

- Insecticide-treated nets;

- Effective case management of malaria illness and anaemia.

Two doses of sulfadoxine-pyrimethamine at monthly intervals after the onset of fetal movements are recommended. In a study in Mali, intermittent preventive treatment with sulfadoxinepyrimethamine significantly reduced the incidence of anaemia, placental infection and low birth weight compared with monthly or weekly chloroquine prophylaxis. ${ }^{10}$

\section{Malaria vaccine}

The initial results of the ongoing phase III clinical trial of the new malaria vaccine, RTS,S (B-cell protein + T-cell epitope+ hepatitis $\mathrm{B}$ surface antigen) have recently been released and are extremely promising. ${ }^{11}$ The vaccine consists of $25 \%$ protein and $75 \%$ hepatitis B surface antigen. The RTS vaccine reduced clinical episodes of malaria and severe malaria by approximately half during the 23 months after vaccination in children 5-17 months of age. Fifteen thousand children in two age categories, between 6-12 weeks of age and 5-17 months of age, were enrolled in the study. During the 14 months after vaccination, the incidence of first episodes of clinical malaria in the older age category was 0.32 episodes per person-year in the vaccine group and 0.55 episodes per person-year in the control group, with an efficacy of $50.4 \%$ (95\% CI 45.8-55.8) in the intention-to-treat population and $55.8 \%$ (95\% CI 50.6-60.4) in the per-protocol population. Vaccine efficacy against severe malaria was $45.1 \%$ in the intention-to-treat population and $47.3 \%$ in the per-protocol population. (The per-protocol population included all participants who received three doses of study vaccine while the intention-to-treat group received one dose of study vaccine.)

The vaccine has the potential to have an important effect on the burden of malaria in Africa. Countries such as Ghana have already started some preparatory discussions to include the vaccine into the country's Expanded Programme on Immunisation.

\section{CLINICAL FEATURES}

The clinical presentation depends on the endemicity of the region. Pregnant women in holoendemic regions (areas with high rates of infection in the community with more than $60 \%$ of children less than 5 years of age infected) are usually asymptomatic because of acquired partial immunity, but the mother is still at an increased risk for anaemia and having a low birth weight baby. ${ }^{12,13}$ In these areas iron deficiency provides some protection against placental malaria. A case-control study in pregnant Malawian women showed that iron-deficient multigravidas were less likely to have placental malaria $(P=0.002) .{ }^{14}$

Certain genetic conditions such as sickle cell trait have also demonstrated some resistance to malarial infection, particularly falciparum malaria. Sickle cell trait has been shown to be $90 \%$ protective against severe and complicated malaria and $60 \%$ protective against clinical malaria requiring hospital admission. ${ }^{15,16}$ The sickle cell trait has also been shown to reduce the death rate from malarial disease in infants between the ages of two and 16 months in endemic areas. ${ }^{17}$ The mechanisms of protection are accelerated sickling of parasitized sickle cells and impaired growth of the parasite in deoxygenated sickle cells. ${ }^{18}$ The increased sickling promotes the removal of infected cells from the circulation.

HIV-positive pregnant women in endemic regions have a greater risk of malarial infection and increased parasite densities with both placental and peripheral infection than HIV-negative women. ${ }^{19} \mathrm{HIV}$-infected women are also more likely to present with symptomatic malaria. ${ }^{20}$

For women residing in mesoendemic regions (areas where transmission is lower and less than $20 \%$ of children less than 5 years of age are affected) or non-immune women who have travelled recently to a malaria area, symptoms and signs may vary from mild febrile illness to severe disease with multiorgan failure. Symptoms may present as early as seven days after exposure; however, in most cases there is a 10-21 day incubation period after being bitten by a mosquito. ${ }^{21} \mathrm{~A}$ high index of suspicion should be entertained in the following circumstances:

- Recent travel history to malaria regions;

- Fever, headache, myalgia, diarrhoea, nausea and vomiting;

- Unexplained thrombocytopaenia;

- Septic miscarriage.

Severe malaria poses a serious risk to both the mother and the fetus. Clinical features include prostration, impaired level of consciousness, respiratory distress, pulmonary oedema, convulsions, shock and abnormal bleeding. ${ }^{22}$

\section{DIAGNOSIS}

The WHO recommends that all cases of suspected malaria be confirmed with a diagnostic test prior to treatment. ${ }^{2}$ The gold standard for diagnosis of malaria is visualization of malarial parasites in peripheral blood by microscopy. The parasite count and the type of species are determined. A negative smear does not exclude the diagnosis and serial smears at 4-6-hour intervals are sometimes necessary to confirm infection. In a febrile patient, three negative smears 12-24 hours apart rule out the diagnosis of malaria. ${ }^{22}$

Rapid diagnostic tests such as the immunochromatographic assays may be used in health facilities where microscopy is not immediately available. Rapid tests are less sensitive than blood film and most tests are only able to detect $P$. falciparum. A result obtained from a rapid test should be confirmed with a blood film, which should always be prepared, even if not 
immediately read. ${ }^{22}$ The rapid test should not be used to monitor disease as it may remain positive for several weeks, even after successful treatment. ${ }^{21}$

\section{TREATMENT}

Pregnant women, especially in the second and third trimesters, have an increased risk of developing severe malaria and have a greater risk of dying than non-pregnant individuals. ${ }^{21}$ Once the diagnosis of malaria is confirmed, a clinical examination and additional laboratory tests should be performed to determine if the patient has severe complicated or uncomplicated malaria. Clinical features of complicated malaria include impaired level of consciousness, respiratory distress, pulmonary oedema, convulsions, shock, abnormal bleeding, jaundice and haemoglobinuria. Patients with a parasite count of more than $2 \%$ should also be treated for severe malaria. ${ }^{22}$ Other laboratory findings suggestive of severe malaria include severe anaemia $(\mathrm{Hb}<8 \mathrm{~g} / \mathrm{dL})$, thrombocytopaenia, hypoglycaemia, acidosis $(\mathrm{pH}<7.3)$ and renal impairment.

Uncomplicated malaria is defined as a parasite count $<2 \%$, without complicating features.

The WHO recommends the following treatment protocol: ${ }^{23}$

\section{Uncomplicated P. Falciparum malaria: admit to hospital:}

\section{First trimester:}

- Quinine plus clindamycin to be given for seven days or artesunate plus clindamycin for seven days if this treatment fails;

- Artemisinin-based combination therapy (ACT) is indicated only if this is the only treatment immediately available or if treatment with seven-day quinine plus clindamycin fails or there is uncertainty of compliance with a seven-day treatment.

\section{Second and third trimesters:}

- ACTs known to be effective in the country/region or artesunate plus clindamycin for seven days or quinine plus clindamycin for seven days.

\section{Lactating women:}

- Lactating women should receive standard antimalarial treatment (including ACTs) except for dapsone, primaquine and tetracyclines.

Treatment of uncomplicated $P$. vivax, $P$. ovale and $P$. malariae malaria:

- Oral chloroquine. Primaquine should not be used in pregnancy.

\section{Treatment of severe malaria:}

- Severe malaria is a medical emergency. After rapid clinical assessment and confirmation of the diagnosis, full doses of parenteral antimalarial treatment should be started without delay with the most effective antimalarial available;

- Artesunate (intravenous or intramuscular)

$\bigcirc$ Intravenous quinine is an acceptable alternative if parenteral artesunate is not available.
- Parenteral antimalarials should be used for a minimum of 24 hours. Thereafter treatment may be completed by giving a course of:

\section{An ACT}

$\bigcirc$ Artesunate plus clindamycin

$\bigcirc$ Quinine plus clindamycin.

(Doxycycline should be avoided in pregnancy.)

The South African Department of Health recommends the following management principles in cases of severe malaria: ${ }^{21}$

(1) Admit patient to a high dependency or intensive care unit;

(2) Monitor fluid balance carefully;

(3) A rapid initial blood glucose level followed by repeated levels every 4-6 hours are essential. Hypoglycaemia is common if the patient is receiving quinine and may be refractory to glucose administration;

(4) Regular monitoring of temperature, respiratory rate, blood pressure and level of consciousness;

(5) Look for and manage any associated infections. If indicated, bacterial meningitis should be excluded by lumbar puncture;

(6) Reduce fever by tepid sponging and paracetamol;

(7) Transfuse if haemoglobin is less than $6 \mathrm{~g} / \mathrm{dL}$ or if anaemia is associated with haemodynamic compromise;

(8) Laboratory tests: regular monitoring of haemoglobin, urea, electrolytes, acid-base status and liver function;

(9) Parasite count should be done daily, and at 72 hours the level of parasitaemia should have dropped by $25 \%$;

(10) If indicated, early dialysis is recommended and may be life-saving;

(11) Treat seizures promptly;

(12) Oxygen, furosemide and assisted ventilation are indicated for respiratory failure.

\section{EFFECTS OF MALARIA ON FETAL AND NEONATAL OUTCOME}

Acute symptomatic malaria and maternal pyrexia are associated with the complications of preterm labour, miscarriage, fetal acidosis, fetal heart rate abnormalities and intrauterine fetal demise.

In endemic areas perinatal outcome is influenced by several factors including maternal parity, HIV status and maternal iron levels. Higher parasite counts and placental infection are encountered more commonly in primgravid and HIV-positive mothers. ${ }^{24} P$. falciparum infections peak between 13 and 18 weeks gestation and it is believed that the malaria parasite impairs trophoblast invasion within the placenta leading to vascular dysfunction and subsequent growth restriction. The inflammatory process and the release of cytokines within the placenta also affects the functional ability of the placenta, thus contributing further to growth restriction. ${ }^{25}$ Recent studies have shown a correlation between placental malaria and the development of hypertension in pregnancy. ${ }^{26}$

Fetal growth restriction is a risk factor for both neonatal and infant mortality. Placental malarial infection and fetal growth restriction also adversely affect postnatal growth and development. In a Malawian study, placental or peripheral infection at delivery was an independent risk factor for slow growth, poor weight gain and failure to thrive in the first year of life. ${ }^{27}$ 
Congenital malaria affects $0.3 \%$ of infants of infected mothers in immune areas and $1-4 \%$ of infants from non-immune areas. Clinical features include fever, irritability, feeding difficulties, jaundice, anaemia and hepatosplenomegaly. Congenital malaria should be considered as an important differential diagnosis of neonatal sepsis. The recommended treatment for children less than one year of age is quinine plus clindamycin. ${ }^{21}$

\section{CONCLUSION}

Malaria is a complex parasitic disease that continues to be a global health challenge. Pregnant women are more susceptible to malaria and have greater risks of developing severe disease with birth complications. Global efforts to prevent malaria are having some impact on public health, and deaths due to malaria are decreasing in some regions of the world. ${ }^{2}$ This momentum must continue and must be sustained if we are to reach the targets set for malarial control by 2015 .

\section{DECLARATIONS}

Competing interests: None.

Funding: None.

Guarantor: Priya Soma-Pillay.

Contributorship: Priya Soma-Pillay researched the literature and wrote the first draft of the manuscript. Both authors reviewed and edited the manuscript and approved the final version.

\section{Acknowledgements: None.}

\section{REFERENCES}

1 Dellicour S, Tatem AJ, Guerra CA, Snow RW, ter Kuile FO. Quantifying the number of pregnancies at risk of malaria in 2007: a demographic study. PLoS Med 2010;7:1-10

2 WHO. World Malaria Report. See www.who.int/malaria/ world_malria_report_2010/en/index.html. (last checked 15 June 2011)

3 van Eijk AM, Hill J, Alegana VA, et al. Coverage of malaria protection in pregnant women in sub-Saharan Africa: a synthesis and analysis of national survey data. Lancet Infect Dis 2011;11:190-207

4 Morris K. Filling the gaps to tackle malaria in pregnancy. Lancet Infect Dis 2011;11:167-8

5 McGregor IA, Wilson ME, Billewicz WZ. Malaria infection of the placenta in the Gambia, West Africa; its incidence and relationship to stillbirth, birthweight and placental weight. Trans $R$ Soc Trop Med Hyg 1983;77:232-44

6 Salanti A, Staalsoe T, Lavsten T, et al. Selective upregulation of a single distinctly structured var gene in chondroitin sulphate A-adhering Plasmodium falciparum involved in pregnancy-associated malaria. Mol Microbiol 2003;49:179-91
7 Beeson JG, Rogerson SJ, Elliott SR, Duffy MF. Targets of protective antibodies to malaria during pregnancy. I Infect Dis 2005;192:1647

8 Staalsoe T, Shulman CE, Bulmer JN, et al. Variant surface antigen-specific IgG and protection against clinical consequences of pregnancy-associated Plasmodium falciparum malaria. Lancet 2004;363:283

9 WHO. Malaria in Pregnancy. See www.int/malaria/high_risk_groups_/ pregnancy/en/index.html (last checked 20 July 2011)

10 Kayentao K, Kodio M, Newman RD, et al. Comparison of intermittent preventive treatment with chemoprophylaxis for the prevention of malaria during pregnancy in Mali. J Infect Dis 2005;191:109

11 The RTS,S Clinical Trials Partnership. First results of phase 3 trial of RTS,S/ASO1 Malaria vaccine in African children. NEJM 2011;365:1863-75

12 Uneke CJ. Diagnosis of Plasmodium falciparum malaria in pregnancy in sub-Saharan Africa: the challenges and public health implications. Parasitol Res 2008;102:333-42

13 Brabin BJ, Ginny M, Sapau J, et al. Consequences of maternal anaemia on outcome of pregnancy in a malaria endemic area in Papua New Guinea. Ann Trop Med Parasitol 1990;84:11

14 Senga EL, Harper G, Koshy G, Kazembe PN, Brabin BJ. Reduced risk for placental malaria in iron deficient women. Malaria J 2011;10:47

15 Hill AV, Allsopp CE, Kwiatkowski D, et al. Common west African HLA antigens are associated with protection from severe malaria. Nature 1991;352:595

16 Willcox M, Bjorkman A, Brohuit J. Falciparum malaria and beta-thalassaemia trait in northern Liberia. Ann Trop Parasitol 1983;77:335

17 Aidoo M, Terlouw DJ, Kolczak MS, et al. Protective effects of the sickle cell gene against malaria morbidity and mortality. Lancet 2002;297:2220

18 Luzzatto L, Nwachuku-Jarrettt ES, Reddy S. Increased sickling of parasitized erythrocytes as mechanism of resistance against malaria in the sickle-cell trait. Lancet 1970;1:319

19 Briand V, Badaut C, Cot M. Placental malaria, maternal HIV and infant morbidity. Ann Trop Paediatrics 2009;29:71-83

20 van Eijk AM, Ayisi JG, ter Kuile FO, et al. HIV increases the risk of malaria in women of all gravidities in Kisumu, Kenya. AIDS 2003;17:596-603

21 Department of Health, Republic of South Africa. Guidelines for the Treatment of Malaria in South Africa. See http://www.doh.gov.za/docs/index.html (last checked 28 June 2011)

22 Lalloo DG, Shinadia D, Pasvol G, et al. UK malaria treatment guidelines. J infect 2007;54:111-21

23 World Health Organisation. Guidelines for the Treatment of Malaria. 2nd edn Geneva: World Health Organisation, 2010. See www.who.int/malaria/ publications/atoz/0789241547925/en/index.html. (last checked 15 June 2011)

24 Nnaji GA, Okafor CI, Ikechebelu JI. An evaluation of the effect of parity and age on malaria parasitaemia in pregnancy. J Obstet Gynaecol 2006;26:755-8

25 Umbers AJ, Aitken EH, Rogerson SJ. Malaria in pregnancy: small babies, big problem. Trends Parasitol 2011;27:168-75

26 Ndao CT. Placental malarial infection as a risk factor for hypertensive disorders during pregnancy in Africa: a case-control study in an urban area of Senegal, West Africa. Am J Epidemiol 2001;170:847-53

27 Kalanda BF. Catch-up growth in Malawian babies, a longitudinal study of normal and low birth weight babies born in a malarious endemic area. Early Hum Dev 2005;81:841-50 